\title{
Estimation of the Error in Measuring the Coordinates of Vibrating Objects Using Laser Coordinate Measuring Systems. General Provisions
}

\author{
Vladimir Fedyaev ${ }^{1}$, Peter Lyudogovsky ${ }^{2}$, and Maria Komkova, ${ }^{2, *}$ \\ ${ }^{1}$ Institute of Mechanics and Engineering of FIC KazanSC of RAS, 420111, Kazan, Russia \\ ${ }^{2}$ Kazan National Research Technical University named after A.N. Tupolev-KAI, 420111, Kazan, \\ Russia
}

\begin{abstract}
The issues of assessing the measurement error using laser coordinate measuring systems (LCMS) of the geometric parameters of vibrating, and also vibrating objects are considered. At the same time, attention is focused on their time-average values. In a number of special cases, calculated relations are given to estimate the average errors in measuring the amplitude and frequency of vibrations of the objects under consideration, their geometric parameters, such as the distance to the laser tracker, coordinates of points on the surface, and other indicators. It is noted that these ratios were obtained for the first time, therefore, in order to increase their reliability, it is possible to introduce them into the corresponding dependences of correcting coefficients and functions.
\end{abstract}

\section{Introduction}

Currently, in the aerospace industry, mechanical engineering, shipbuilding, nuclear power, and in many other sectors of the economy to perform high-precision measurements of the dimensions of objects, geometric parameters of assembled parts of complex structures, control the process of their docking, etc. laser coordinate measuring systems (LCMS) based on trackers are increasingly used [1-4]. In real conditions of using these systems, the measurement accuracy is influenced by a set of both internal (errors of drives of trackers, interferometers, absolute rangefinders, spherical reflectors) [5-8], and external (composition, pressure, temperature, air humidity [7, 9-14]) factors. External factors should also include in the general case vibrations of the devices involved, measured objects, environmental indicators [15-23]. passing vehicles, gusts of wind, sources of turbulent disturbances of air flows, temperature, etc. Since ensuring the required accuracy, including taking into account external factors, is mandatory for any measurements, the study of the influence of these factors on the measurement accuracy seems to be very relevant.

\footnotetext{
* Corresponding author: matrjshka@mail.ru.
} 


\section{Research method}

Considering the fact that relatively few works [20-23] are devoted to the study of the influence on the accuracy of measurements using LCMS of the geometric parameters of objects in the presence of vibrations, while they can lead to significant errors, let us consider the issues of assessing these errors from a general point of view.

The main device of LCMS is a laser tracker (for example, Radian API), which includes an interferometer, an absolute rangefinder, optical system servo drives, and protractors. The laser beam emitted by the rangefinder, hitting the center of the spherical reflector, returns back to the lens of the device to the rangefinder, which measures the distance from the laser tracker to the center of the spherical reflector [1]. Using this distance, measured horizontal and vertical angles, the coordinates of the center of the spherical reflector are found, i.e. the position in space of the control point on the object's surface. Thus, in general, when carrying out measurements, objects and measuring instruments, the environment are involved, all of them can vibrate. Since these vibrations are very diverse, the measurement objects are quite complex, we will accept the following simplifying assumptions.

1. The main initial causes of vibrations of measurement objects, measuring instruments (trackers) are vibrations of supports on which they are located, as well as directly themselves, due to the action of external factors (mechanical, thermal).

2. When taking measurements indoor, due to the relatively small distances between objects and measuring instruments, it is considered that the supports of both of them oscillate synchronously, as a whole; the indicators of vibrations of the environment (air) near them, along the path of the laser beam are the same.

3. Mutual slippage of support structures (slipways) of objects, legs of tripods of trackers with the surfaces of corresponding supports, support pads are small and cannot be taken into account.

4. The forced oscillations of the components of the systems under consideration are distinguished as the main ones, as well as, if necessary, natural oscillations.

Determination of the amplitude, frequency, and other parameters of the oscillations of these components is carried out by measurements either directly in production conditions or in laboratory, in the course of performing the corresponding experiments. An alternative to these methods is the calculation of the required indicators using software packages, analytical methods and dependencies, widely presented in the scientific and technical literature [24-26, etc.]

The estimation of the error of the obtained results of measurements, calculations (computational experiments) is significantly complicated by the fact that oscillations and vibrations proceed in time. Moreover, the amplitude we have identified, the frequency of oscillations can also change over time. Therefore, keeping in mind engineering practice, by the estimation of the error of the results of measurements and calculations we mean the values averaged over time.

In particular, when, in the course of natural or laboratory experiments, a slightly deformable object is rigidly fixed on the surface of an oscillation source with a regulated amplitude $A_{o}$ and frequency $\Omega_{o}$, then

$$
\Delta A=A_{k}-A_{o}, \Delta \Omega=\Omega_{k}-\Omega_{o} .
$$

Here $A_{k}, \Omega_{k}$ are the average controlled (measured) amplitude, vibration frequency of certain geometric parameters of the object under study. The differences $\Delta A, \Delta \Omega-$ can be considered, of course, rather conditionally, the average errors of measurements of these quantities. 
Another way to estimate the considered errors is as follows. Suppose that there are results of measurements (calculations) of the dynamics of oscillations of a certain point located on the object, described by the relation:

$$
y=f(\tau)=a \sin (\omega \tau) .
$$

Here $y$ characterizes the displacement of a point relative to the middle position $y=0$, $\tau$ - time; $a=a(\tau), \omega=\omega(\tau)$ - respectively, the amplitude and frequency of timedependent oscillations. Further, we divide the measurement time $\tau_{k}$ into a number of cycles characterized by the period

$$
T=T(\tau)=2 \pi / \omega(\tau),
$$

moreover, each of these cycles will be considered as a separate test. With such an interpretation of experiments in accordance with the theory of errors [27], the errors $\Delta a_{i}$, $\Delta \omega_{i}$ of the amplitude and vibration frequency measurements for each of the cycles are easily found:

$$
\Delta a_{i}=a_{i}-a_{o}, \Delta \omega_{i}=\omega_{i}-\omega_{o} .
$$

Here $i$ is the cycle number; $a_{o}, \omega_{o}$ are the average amplitude and frequency of oscillations for the entire experiment $\tau_{k}$.

Since these values, in general, depend on time, averaging them, we obtain the average errors:

$$
\Delta a=\frac{1}{k} \sum_{i=1}^{k} \Delta a_{i}, \Delta \omega=\frac{1}{k} \sum_{i=1}^{k} \Delta \omega_{i},
$$

where $k$ is the number of oscillation cycles per time $\tau_{k}$.

It is known that when averaging of function $y=f(\tau)$ (2) over time $\tau_{k}$ describing oscillations, vibrations of a point near the position $y=0$, the integral

$$
y_{o}=\frac{1}{\tau_{k}} \int_{0}^{\tau_{k}} f(\tau) d \tau
$$

may not be zero. This means that, on average, this point is displaced relative to its position $y=0$ by an amount $y_{o}$. Such a possibility appears when in (2) the amplitude $a$, frequency $\omega$, either separately or simultaneously, depend on time. In the simplest case, functions $a=a(\tau), \omega=\omega(\tau)$ can be approximated by linear relations:

$$
a=a_{o}+b_{o} \tau, \omega=\omega_{o}+\beta_{o} \tau,
$$

where $a_{o}, b_{o}, \omega_{o}$ and $\beta_{o}$ are determined as a result of processing experimental data using, for example, the least squares method, or theoretically in the presence of appropriate analytical dependencies. If over time $\tau_{k}$ the frequency of oscillations changes weakly, $\beta_{o}=0 ; \omega_{o}$, period $T_{o}=2 \pi / \omega_{o}$ are constant values, then, without going into details, for the evaluation $y_{o}$ we obtain the expression: 


$$
y_{o}=-0.16 T_{o} \sum_{i=1}^{k-1} \bar{a}_{i} /(k-1) .
$$

Here, as before, $k$ is the number of oscillation cycles of a point during time $\tau_{k}$; $\bar{a}_{i}=d a(\tau) / d \tau$ at $\tau=i T_{o}, i$ - cycle number $(i=1,2, \ldots, k-1)$.

In the second case, when the amplitude of oscillations changes little in time, $b_{o}=0, a_{o}$ is constant, but the frequency of oscillations changes, but insignificantly, after a series of transformations we find

$$
y_{o}=2 a_{o} \beta_{o} / \omega_{o}^{2} .
$$

Hence it follows that in the case under consideration, the average displacement of the oscillating point is proportional to the amplitude of the oscillations $a_{o}$, the coefficient $\beta_{o}$ characterizing the change in the oscillation frequency over time is inversely proportional to the value $\omega_{o}^{2}$.

The last, third case is undoubtedly more complicated; nevertheless, the calculated ratios, in principle, can be obtained. However, in practice, the situation typical for this case is least of all. At the same time, if we assume that there is an additivity of the influence on the value of the average bias of both factors considered above, then in this case

$$
y_{o}=-0.16 T_{o} \sum_{i=1}^{k-1} \bar{a}_{i} /(k-1)+2 a_{o} \beta_{o} / \omega_{o}^{2} .
$$

Provided that the geometric parameters of vibrating objects are mathematically described by relations of the form (2), the obtained dependences (4) - (6), in our opinion, can be used to estimate the average error of their measurements.

\section{Conclusions}

Thus, we have proposed expressions (1), (3), we emphasize, for evaluating the time-averaged errors in measuring the amplitudes and frequencies of oscillations; formulas (4) - (6), allowing for a nonlinear law of oscillations of geometric indicators (distance from the tracker, coordinates of individual points, etc.) of the objects under consideration to estimate the average errors of their measurements. The approaches presented in the work, which make it possible to obtain the named relations, are new, we admit that they are controversial. If it is necessary to clarify these ratios, you can enter correction coefficients or functions.

\section{References}

1. V.V. Yakunin, D.G. Vikoruk, Laser coordinate-measuring systems API Tracker 3, Bulletin of the metrologist, 2, pp. 13-15 (2009)

2. V.N. Grishanov, A.A. Oynonen, Up-to-date laser measuring systems in the production cycle of space technique, Bulletin of the SSAU, 1, pp. 24-35 (2012)

3. S.N. Shaposhnikov, O.S. Efremkin, Geometrics control of high-accuracy benches for traffic control systems detecting elements of spacecraft, Izvestia of Samara, Scientific Center of the Russian Academy of Sciences, 20 (4), pp. 55-60 (2018)

4. V.V. Petrov, V.O. Medyannikov, E.V. Kraev, The use of a laser tracker for monitoring the position of the stator wedges of a large hydrogenerator, SHEM-70 years in hydropower: Scientific and technical. Sat. - SPb, pp. 213-219 (2012) 
5. Z. Hejun, M. Junjie, Z. Jun, Multi Parameter Error Model and Calibration of Laser Tracker, Chinese Journal of Scientific Instrument, 41 (9), pp. 20-30 (2020)

6. Brochure of the laser tracker API Radian [Electronic resource]. URL: https://nevatec.ru/wp-content/uploads/2017/02/radianpropluscore_web_ip54_vp2.pdf (date accessed: 07/05/2021).

7. ASME B89.4.19-2006. Performance Evaluation of Laser-Based Spherical Coordinate Measurement Systems, 42 p (2006)

8. L. Wan-li Liu, Jian-fei Ouyang, Xing-hua Qu, Effect of Incident Laser Beam Angle Varying on Cube Corner Retro-Reflector Measurement Accuracty, Optics and Precision Engineering, 17 (2), pp. 286-291 (2009)

9. V.E. Zuev, Propagation of laser radiation in the atmosphere (Radio and communication, 1981)

10. I.N Matveev, V.V. Protopopov, I.N. Troitsky, N.D. Ustinov, Laser location, (Mechanical engineering, 1984)

11. K.Boren, D. Huffman, Absorption and scattering of light by small particles, (Mir, 1986)

12. G.J. Siddall, R.R. Baldwin, Development in Laser Interferometry for Position Sensing, Precision Engineering, 6 (4), pp. 175-180 (1984)

13. B. Edlen, The Refractive Index of Air, Metrologia, 2 (2), pp. $71-79$ (1966)

14. K.P. Birch, M.J. Downs, Correction to the Updated Edlen Equation for the Refractive Index of Air, Metrologia, 31 (4), pp. 315-316 (1994)

15. I.I. Blekhman, G.Yu. Janelidze, Vibration Displacement, (Nauka, 1964)

16. Yu.V. Kiryanov, On the vibrational movement of the horizontal circle of theodolites, Geodesy and aerial photography, 1, pp. 125-131 (1992)

17. I.Yu. Vasyutinsky, Influence of vibration on the accuracy of hydrostatic leveling, Geodesy and aerial photography, 5, pp. 43-50 (1977)

18. Yu.V. Kiryanov, N.M. Komar. The effect of vibration on the stability of tripods, Geodesy and aerial photography, 4, pp. 112-117 (1984)

19. V.N. Naidenko, H.K. Yambaev, Features of the influence of vibration on optical theodolites. Geodesy and aerial photography, 3, pp. 113-118 (1987).

20. G.A. Ustavich, A.V. Koshelev, O. P. Poshivailo, On the influence of vibration on lightrange measurements, Geodesy and cartography, 6, pp. 8-10 (1998)

21. A.A. Beshtr, A.V. Ivanov, Research of the tripod vibration effect on the terrestrial laser scanner measurements accuracy, Sb. materials of the VI Int. scientific. Congr. "GEOSiberia-2010", 1 (3), pp. 90-94 (2010)

22. A.A. Beshr, N.M. Ryabova, V.G. Salnikov, M.R. Rahimberdina, Studying the effect of vibration of "tripod-level" system on the measurements accuracy of digital levels, Sb. materials of the VI Int. scientific. Congr. "GEO-Siberia-2010", 1 (1), pp. 28-32 (2010)

23. A.I. Kuzmich, S.S. Bogdanov, Research on the influence of vibrations on modern digital levels, Geodesy and aerial photography, 1 (2014)

24. S.P. Timoshenko, Theory of Oscillations in Engineering (Moscow - Leningrad, 1932)

25. DV Weinberg, G.S. Pisarenko, Mechanical vibrations and their role in technology, (Nauka, 1965)

26. A. Kobayashi, Experimental Mechanics, 3 (Mir, 1990)

27. I. N. Bronstein, K.A. Semendyaev, Handbook of Mathematics for Engineers and University Students (Nauka, 1986) 